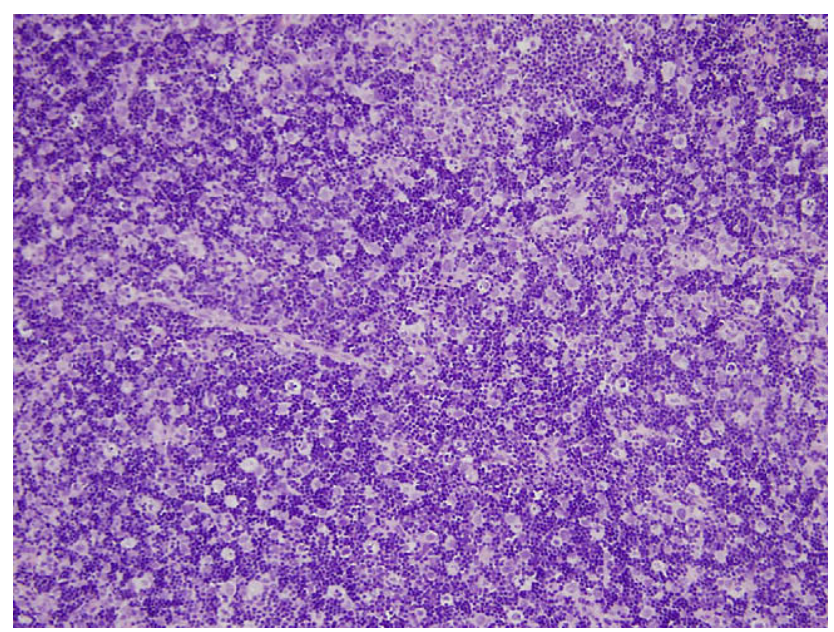

FIGURE 2. Tumor is characterized by large, coarse lobules of tumor with delicate septa, resembling lobular architecture of thymus cortex. Neoplastic tumor cells with polygonal nuclei are intermixed with lymphocytes (hematoxylin and eosin stain; original magnification $\times 200$ ). Histologic analysis was consistent with type B2 thymoma.

blood supply as a result of its rapid enlargement was among the causes of the necrosis. Ten cases of spontaneous regression of thymoma have been reported in the Japaneselanguage literature. ${ }^{3}$ In all cases, the bulk of each tumor was necrotic, with regression being thought to be related to the necrosis. Most thymomas with spontaneous regression are encapsulated, and their prognoses are good.

Apparent spontaneous regression of various malignant neoplasms has been described, although as an unusual phenomenon. Most of these patients had renal cell carcinoma, malignant melanoma, or neuroblastoma. Immunologic factors have been described as playing a significant role. ${ }^{4}$ The spontaneous regression of thymoma has rarely been reported.

We report here the case of a patient with an anterior mediastinal thymoma. The mediastinal thymoma regressed spontaneously 3 years after diagnosis without treatment. We did not obtain specimens to determine the pathologic findings after tumor regression. In this case, the spontaneous regression was seen as the complete disappearance of the tumor on radiologic imaging, with no observed intervening decrease in tumor size. This is different from previous reports. Initially, the patient did not have any symptoms or pleural effusion on follow-up chest radiography. The phenomenon of spontaneous regression may not be related to rapid enlargement and necrosis, and the actual mechanism of spontaneous regression of this thymoma is not clear. This may be clarified in a collection of similar cases. The regression of an invasive thymoma has been reported after corticosteroid therapy. ${ }^{5}$ Our patient did not receive any medical treatment, however, including steroids. Complete spontaneous regression of mediastinal thymomas is possible. Although the phenomenon is rare, conservative treatment may be an alternative to more aggressive therapy for patients with thymoma who are medically unfit.

\section{References}

1. Kuo T. Sclerosing thymoma-a possible phenomenon of regression. Histopathology. 1994;25:289-91.

2. Okagawa T, Uchida T, Suyama M. Thymoma with spontaneous regression and disappearance of pleural effusion. Gen Thorac Cardiovasc Surg. 2007;55:515-7.

3. Ishibashi H, Ashino Y, Niikawa H, Hosaka T, Hasumi T, Shibuya J, et al. [Spontaneous regression of thymoma; report of a case.] [Japanese]. Kyobu Geka. 2003;56:801-5.

4. Cole WH. Spontaneous regression of cancer: the metabolic triumph of the host? Ann N Y Acad Sci. 1974;230:111-41

5. Barratt S, Puthucheary ZA, Plummeridge M. Complete regression of a thymoma to glucocorticoids, commenced for palliation of symptoms. Eur J Cardiothorac Surg. 2007;31:1142-3.

\title{
Acute myocardial infarction and limb ischemia as manifestation of nonbacterial thrombotic endocarditis
} \author{
Montreal, Quebec, Canada \\ We report a 29-year-old patient who underwent right \\ femoropopliteal bypass 2 years previously for right leg \\ From the Divisions of Cardiac Surgery ${ }^{\mathrm{a}}$ and Cardiology, ${ }^{\mathrm{b}}$ Jewish General Hospital, \\ Montreal, Quebec, Canada. \\ Received for publication March 17, 2008; accepted for publication April 21, 2008. \\ Address for reprints: Jean-François Morin, MD, FRCS, Division of Cardiac Surgery, \\ Jewish General Hospital, A520, 3755 Chemin Côte-Ste-Catherine, Montreal, Que- \\ bec, Canada H3T 1E2 (E-mail: jmorin@surg.jgh.mcgill.ca). \\ J Thorac Cardiovasc Surg 2009; 137:1278-80 \\ $0022-5223 / \$ 36.00$ \\ Copyright (c) 2009 by The American Association for Thoracic Surgery \\ doi:10.1016/j.jtcvs.2008.04.013
}

Naoko Nagano, MD, ${ }^{\mathrm{a}}$ Jean-François Morin, MD, FRCS, ${ }^{\mathrm{a}}$ Dominique Joyal, MD, ${ }^{\mathrm{b}}$ and Lawrence Rudski, MD,

ischemia and was admitted recently to the hospital for acute myocardial infarction secondary to nonbacterial thrombotic endocarditis.

\section{CLINICAL SUMMARY}

A 29-year-old man was admitted to an emergency department with acute onset chest pain. His electrocardiogram showed typical anterior acute myocardial infarction with remarkable ST segment elevations. Emergency coronary angiography was performed, which revealed a complete 

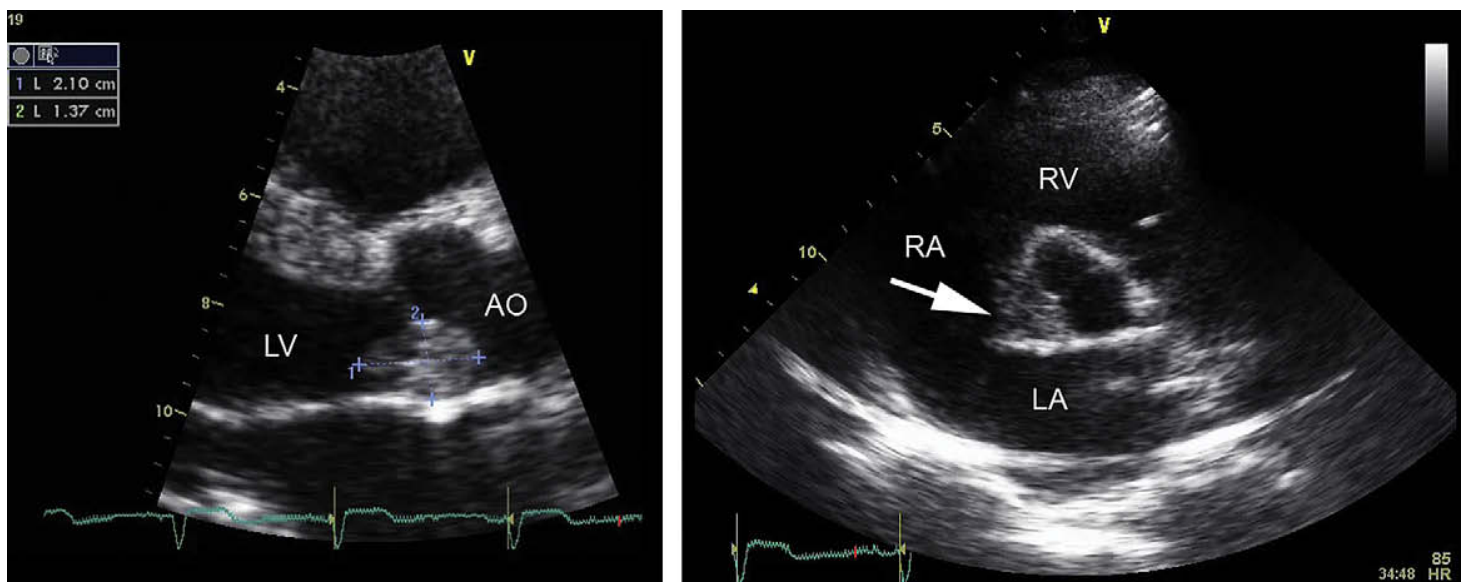

FIGURE 1. Preoperative transthoracic echocardiography. Long-axis view (left). Short-axis view (right). Vegetation (arrow) was seen on the noncoronary aortic leaflet. $L V$, Left ventricle; $A o$, aorta; $R A$, right atrium; $R V$, right ventricle; $L A$, left atrium.

occlusion of the proximal left anterior descending artery. The right and circumflex arteries were entirely normal. After anticoagulation with heparin and abciximab, the occlusion was crossed and the clot was aspirated through an aspiration catheter. The vessel was successfully stented with a good result. The patient's medical history included a big toe amputation because of ischemia and femoropopliteal bypass surgery 2 years ago, at which time Burger disease was diagnosed. He did not have any other medical history except for cocaine use. An echocardiogram was performed the following day, which showed a 21 $\times 14$-mm irregularly shaped mass on the noncoronary leaflet of the aortic valve and a mobile mass measuring $10 \times 6 \mathrm{~mm}$ in the left ventricular outflow tract (Figure 1). Severe aortic regurgitation was also found with a left ventricular ejection fraction of $35 \%$. Because it was highly probable that the mass was a source of thromboembolization, an urgent aortic valve replacement with a $25-\mathrm{mm}$ bileaflet mechanical valve was performed on the fourth day after acute myocardial infarction.

During surgery, the mass was found along the coaptation line on the noncoronary leaflet of the aortic valve. There was some thrombus on the surface of the mass (Figure 2). The aortic valve was tricuspid with an apparently small noncoronary cusp, and there was some thickening at its base. The subsequent coagulation profiles were normal and immunology test results, including lupus anticoagulant antibodies, anticardiolipin antibodies, and rheumatoid factor and cultures, were negative. The pathologic results of the mass and aortic valve found no infectious cause but did show evidence of thrombus. We concluded that the patient's aortic valve lesion was nonbacterial thrombotic endocarditis. His postoperative course was uneventful, and he was discharged with oral anticoagulation therapy.

\section{DISCUSSION}

Nonbacterial thrombotic endocarditis, previously known as marantic endocarditis, is most commonly seen in patients with advanced malignant disease and hypercoagulative state. It is described as a sterile vegetation composed of platelets and fibrin that adhere to valvular structures, most commonly found along coaptation lines, and is susceptible to embolization. ${ }^{1,2}$ In autopsy studies, evidence of embolization was found in approximately $40 \%$ of cases. ${ }^{2}$ In addition to advanced malignant disease, autoimmune disease and connective tissue disorder have been well-known predisposing factors for nonbacterial thrombotic endocarditis. Nonbacterial thrombotic endocarditis occurs both on pathologic valves and on relatively normal valves. ${ }^{3}$ The most frequent underlying lesions include chronic rheumatic heart valve disease or nonspecific abnormality. ${ }^{3}$ Patrick and colleagues $^{2}$ reported a series of 30 patients who underwent valve replacement for nonbacterial thrombotic endocarditis: Eight patients (27\%) had antiphospholipid syndrome; 6 patients $(20 \%)$ had rheumatic heart valve disease; 2 patients

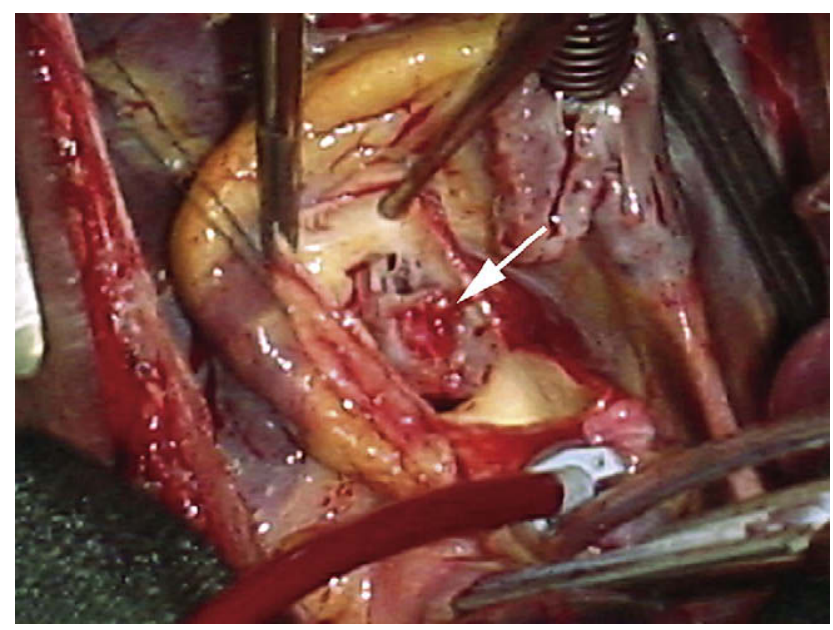

FIGURE 2. An intraoperative photograph was taken from patient's cranial to caudal view. Thrombus and vegetation (white arrow) on the noncoronary aortic leaflet. 
(7\%) had systemic lupus erythematosus; 2 patients $(7 \%)$ had chronic rheumatoid arthritis; 9 patients $(30 \%)$ had other underlying risk factors, such as congenital or degenerative heart valve disease or coagulation problem; and 3 patients $(10 \%)$ had no risk factors. The majority of their patients underwent surgery for valve dysfunction.

When patients can tolerate cardiac surgery with cardiopulmonary bypass, indications for surgery are valve dysfunction, recurrent embolic events, ${ }^{4}$ and mobile vegetation. Removal of vegetation and repair of the valve seem to be feasible if the vegetation is small and localized. Prognosis after valve replacement or repair for nonbacterial thrombotic endocarditis is unknown but depends on the underlying cause.

Even if the previous femoropopliteal bypass was performed with the working diagnosis of Burger disease, it is unlikely because there was no arterial disease in the other leg. Because of the large size of the vegetation plus the possible delay of time between ischemic events, ${ }^{5}$ the patient's limb ischemia was thought to be arterial embolization caused by the vegetation. His noncoronary aortic leaflet was atrophic, which may be a factor in causing nonbacterial thrombotic endocarditis. Despite the patient's young age and good general condition, he should be monitored carefully for possible occult malignant disease.

\section{CONCLUSIONS}

Nonbacterial thrombotic endocarditis should be considered when a patient presents with recurrent embolization in the absence of a history of malignant disease, hypercoagulative state, or atrial fibrillation. Surgical removal of the vegetation is effective to prevent further embolization, and valve replacement or repair may be essential to correct valve dysfunction.

\section{References}

1. Borowski A, Ghodsizad A, Cohnen M, Gams E. Recurrent embolism in the course of marantic endocarditis. Ann Thorac Surg. 2005;79:2145-7.

2. Patrick WE, William DE, Henry DT, Robert DM, Kenton JZ. Surgical pathology of nonbacterial thrombotic endocarditis in 30 patients, 1985-2000. Mayo Clin Proc. 2001;76:1204-12.

3. Kardaras FG, Kardara DF, Rotntoglani DP, Sioras EP, Christopoulou-Cokkinou V, Lolas CT, et al. Acute aortic regurgitation caused by non-bacterial thrombotic endocarditis. Eur Heart J. 1995;16:1152-4.

4. Rabinstein AA, Giavanelli C, Ricci M, Romano JG, Koch S, Forteza AM. Surgical treatment of nonbacterial thrombotic endocarditis presenting stroke. J Neurol. 2005;252:352-5.

5. Callander N, Rapaport SI. Trousseau's syndrome. West J Med. 1993;158: 364-71.

\section{Multiple papillary fibroelastoma with quadricuspid aortic valve}

Reiji Hattori, MD, PhD, ${ }^{\text {a }}$ Chihiro Oishi, MD, Junji Iwasaka, MD, PhD, ${ }^{\mathrm{b}}$ Toshiji Iwasaka, MD, PhD, ${ }^{\mathrm{b}}$ Takayuki Okada, MD,

Hiroyuki Johno, MD, ${ }^{\mathrm{a}}$ Chiharu Enoki, MD, ${ }^{\mathrm{a}}$ Tomohiko Sumida, MD, ${ }^{\mathrm{a}}$ Yoshihisa Nakao, MD, PhD, ${ }^{\mathrm{a}}$ and

Hiroji Imamura, MD, PhD, ${ }^{a}$ Hirakata, Osaka, Japan

Quadricuspid aortic valve (QAV) is a rare congenital anomaly that is one of the causes of aortic valve insufficiency, which often requires aortic valve replacement. Cardiac papillary fibroelastoma $(\mathrm{CPF})$ is also a rare benign cardiac tumor, and there are reports of rare cases of multiple occurrences. We report our experience of successful surgery for a previously unreported condition in a patient with a QAV who had a narrow aortic annulus associated with complications of multiple CPF.

From the Department of Thoracic and Cardiovascular Surgery ${ }^{\mathrm{a}}$ and Division of Cardiology, Second Department of Medicine, ${ }^{\mathrm{b}}$ Kansai Medical University, Hirakata, Osaka, Japan.

Received for publication March 21, 2008; accepted for publication April 20, 2008.

Address for reprints: Reiji Hattori, MD, PhD, Department of Thoracic and Cardiovascular Surgery, Kansai Medical University, 2-3-1, Shinmachi, Hirakata, Osaka, 573-

1191, Japan (E-mail: hattori@hirakata.kmu.ac.jp).

J Thorac Cardiovasc Surg 2009; 137:1280-2

$0022-5223 / \$ 36.00$

Copyright (c) 2009 by The American Association for Thoracic Surgery doi:10.1016/j.jtcvs.2008.04.015

\section{CLINICAL SUMMARY}

A 71-year-old woman underwent a transthoracic echocardiogram as part of a detailed examination for the treatment of peripheral arterial disease, and mobile masses were incidentally found that were attached to the aortic valve. In addition, the aortic valve was a QAV with severe aortic regurgitation, and various sizes of mobile masses were attached to each of the 4 cusps detected by a transesophageal echocardiogram (Figure 1). Moreover, similar mobile masses were also observed in the left ventricular outflow tract (LVOT) and mitral chordae. In November of 2007, the patient underwent an urgent operation. The aortic valve was observed through an oblique aortotomy using a standard technique of cardiopulmonary bypass under cardiac arrest obtained with blood cardioplegia. Various sizes of mobile tumors were attached to each of the 4 cusps on the ventricular side. The aortic valve was carefully excised with the attached tumors. Through observations of the removed aortic valve placed in water, we found that 